\title{
Suspended silicon integrated platform for the long-wavelength mid-infrared band
}

\author{
Alejandro Sánchez-Postigo, Gonzalo Wangüemert-Pérez, Jordi Soler Penadés*, Alejandro Ortega- \\ Moñux, Milos Nedeljkovic*, Robert Halir, Faysal El Mokhtari Mimum, Zhibo Qu*, Ali Z. Khokhar*, \\ Ahmed Osman*, Wei Cao*, Callum G. Littlejohns*, Goran Z. Mashanovich*, Pavel Cheben†, Íñigo \\ Molina-Fernández \\ Universidad de Málaga, Dpto. Ingeniería de Comunicaciones, ETSI Telecomunicación, 29071 Málaga, Spain \\ * Optoelectronics Research Centre, University of Southampton, Southampton, SO17 1BJ, United Kingdom \\ $\dagger$ National Research Council Canada, 1200 Montreal Road, Bldg. M50, Ottawa KIA OR6, Canada
}

Tel: (+34) 952132853,e-mail: asp@ic.uma.es

\begin{abstract}
The atmospheric-transmission window and the fingerprint region of many substances overlaps with the longwave infrared band. This has enabled the emergence of a new path for photonic integrated circuits, which could exploit the potential applications of this wavelength range, including chemical and bio sensing. In this work we review our latest advances in the suspended silicon platform with subwavelength grating lateral cladding at 7.7$\mu \mathrm{m}$ wavelength. Suspended waveguides only require one lithographic etch step and can be specifically designed to maximize sensitivity when used as sensors. Waveguides with propagation loss of $3.1 \pm 0.3 \mathrm{~dB} / \mathrm{cm}$ are demonstrated, as well as bends with less than $0.1 \mathrm{~dB} /$ bend. Suspended waveguides based on shifted Bragg grating lateral cladding are also reported, with propagation loss of $5.1 \pm 0.6 \mathrm{~dB} / \mathrm{cm}$. These results prepare the ground for the development of a platform capable of covering the entire mid-infrared band.
\end{abstract}

Keywords: suspended silicon, mid-infrared, long-wave infrared, subwavelength grating, Bragg.

\section{INTRODUCTION}

The advent of quantum cascade lasers and detectors for the mid-wave infrared (MIR) range has motivated the development of integrated platforms in this spectral band [1,2]. MIR wavelengths overlap with the atmospherictransmission window and the fingerprint region of many organic and inorganic molecules [3]. This opens the possibility of designing new waveguides and devices to both signal processing and sensing applications.

The silicon on insulator (SOI) platform, formed of a stack of silicon, silicon dioxide and silicon layers, has been extensively used in the microelectronics industry since the eighties and is the dominant substrate for silicon photonics [4]. The commercial availability, the compatibility with CMOS manufacturing processes and the maturity reached in the near-infrared (NIR) range could make SOI the most attractive candidate for the development of mid-infrared photonic integrated circuits. However, while silicon waveguides can easily operate with low-loss up to $\lambda \sim 8$ um, silicon dioxide becomes lossy from $\sim 4-\mu \mathrm{m}$ [3]. In [5], the authors leverage SOI waveguides with wide and thick cross sections to achieve low loss up to $\lambda=6 \mathrm{um}$. However, these waveguides are not suitable for sensing applications in the long-wavelength mid-infrared regime.

To take benefit from the entire transparency window of silicon, we propose the use of suspended silicon waveguides. The silicon dioxide is removed with a hydrofluoric (HF) acid solution. Two alternatives have been reported in the literature to accomplish suspension: suspended membranes and suspended waveguides with subwavelength grating lateral cladding.

Suspended membranes are rib waveguides with holes etched at both sides of the silicon core [6]. These holes enable the flow of $\mathrm{HF}$ acid to reach the silicon dioxide without interacting with the fundamental mode propagating through the waveguide core. Two lithographic etch steps are required: i) for defining the rib and ii) for etching the holes.

Alternatively, we proposed suspended silicon strip waveguides with subwavelength grating (SWG) lateral cladding [7]. Here, only one dry etch step is needed, which simultaneously defines the waveguide core and an SWG lattice of holes. The SWG cladding can be modelled as a homogeneous metamaterial with a synthesized refractive index that creates the index contrast required for waveguiding [8,9]. Additionally, the cladding supports the suspended waveguide core. As in membrane waveguides, the holes allow HF removal of silicon dioxide. Figure 1 represents a schematic of our suspended silicon waveguide along with its geometrical parameters. In this work we will review our recent advances in passive devices for this platform. 

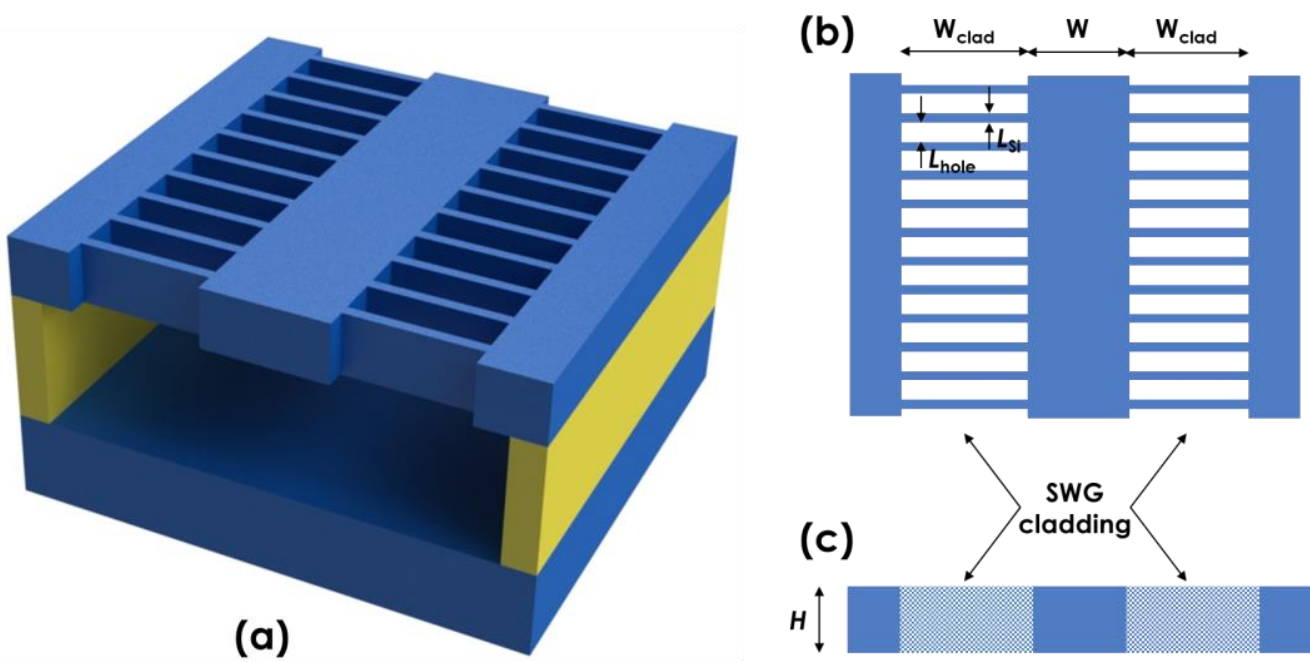

Figure 1. Schematics of a suspended silicon waveguide with subwavelength grating lateral cladding: (a) $3 D$ view of the suspended waveguide with subwavelength grating lateral cladding, (b) top view of the silicon guiding layer and (c) front view of the silicon guiding layer. Blue: silicon; yellow: silicon dioxide.

\section{SUSPENDED SILICON WAVEGUIDES AND BENDS WITH SUBWAVELENGTH GRATING LATERAL CLADDING}

Suspended silicon waveguides must be carefully designed to guarantee single-mode operation and mechanical stability. The former requires that quasi- $\mathrm{TE}_{01}$ and quasi-TE $\mathrm{TE}_{10}$ modes, if supported, be leaked to the substrate and lateral silicon, respectively, while the quasi-TE 00 mode is guided with low loss. The geometrical parameters that need to be designed are summarized in Table 1, indicating whether high $(\uparrow)$ or low $(\downarrow)$ values are required for each requirement. Mechanical robustness requires a light waveguide core, a reduced mechanical torque and a lateral cladding preventing the waveguide core from collapsing. Table 2 shows the geometrical dimensions that increase the structural robustness.

Table 1. Design requirements for single-mode operation.

\begin{tabular}{|l|c|c|c|c|}
\hline Requirement & $H$ & $W$ & $W_{\text {clad }}$ & $L_{\text {Si }}$ (with constant $\Lambda<\Lambda_{\text {Bragg }}$ ) \\
\hline Reduced fundamental mode substrate leakage & $\uparrow$ & $\uparrow$ & N/A & $\uparrow$ \\
\hline Reduced fundamental mode lateral leakage & $\uparrow$ & $\uparrow$ & $\uparrow$ & $\downarrow$ \\
\hline Increased high-order mode substrate leakage & $\downarrow$ & $\downarrow$ & N/A & $\downarrow$ \\
\hline Increased high-order mode lateral leakage & $\downarrow$ & $\downarrow$ & $\downarrow$ & $\uparrow$ \\
\hline
\end{tabular}

Table 2. Design requirements for mechanical stability.

\begin{tabular}{|l|c|c|c|c|}
\hline Requirement & $H$ & $W$ & $W_{\text {clad }}$ & $L_{\text {Si }}$ (with constant $\Lambda<\Lambda_{\text {Bragg }}$ ) \\
\hline Light waveguide core & $\downarrow$ & $\downarrow$ & N/A & N/A \\
\hline Reduced torque & $\downarrow$ & $\downarrow$ & $\downarrow$ & N/A \\
\hline Robust cladding & N/A & N/A & N/A & $\uparrow$ \\
\hline Easy flow of HF acid solution & $\downarrow$ & N/A & N/A & $\downarrow$ \\
\hline
\end{tabular}

As it can be seen in Table 1, it is not possible to design a waveguide to simultaneously minimize leakage for the fundamental mode and maximize leakage for the second-order mode. Besides, the geometrical parameters for low-loss fundamental mode are not compatible with mechanical stability (Table 2). At the same time, the period $\Lambda$ must be kept below the Bragg regime, i.e. $\Lambda<\Lambda_{\text {Bragg }}$ [9]. For a central wavelength of $\sim 7.7 \mu \mathrm{m}$, the waveguides were judiciously designed assuming the cladding behaves as a homogeneous material with a synthesized refractive index. Final dimensions are indicated in Table 3.

The fabrication process was as follows. After cleaning, SOI wafers with a $\mathrm{SiO}_{2}$ layer of $3 \mu \mathrm{m}$ are spin-coated with ZEP520-A photoresist. The lay-out is defined in the photoresist with e-beam lithography. An inductively coupled plasma (ICP) tool is utilized to etch the holes of the suspended waveguides in the upper silicon layer. This step leaves the underlying silicon dioxide exposed. The sample is finally dipped into a tank with 1:7 HF acid solution, which suspends the waveguides, and rinsed. The duration of the dipping is critical: if the sample is not enough time in contact with the HF solution, remains of silicon dioxide can increase propagation loss; on the other hand, if dipping time is excessive, silicon thickness and width can be affected by the acid. Thus, after 
several experiments, we left samples two and a half hours inside the HF tank. Figure 2(a) shows a SEM image of a fabricated suspended silicon waveguide.

For characterization, we utilized a continuous-wave quantum cascade laser (QCL) emitting at $\sim 7.7 \mu \mathrm{m}$. Light was coupled into the chip using chalcogenide optical fibers (mode field diameter of $~ 29.5 \mathrm{um}$ ) using suspended grating couplers. Power transmitted through the waveguides was measured with a $\mathrm{LN}_{2}$-cooled $\mathrm{HgCdTe}$ photodetector. To improve the signal-to-noise ratio, light was passed through a chopper wheel, whose turning frequency was used as a reference for a lock-in amplifier connected to a computer.

As shown in Table 3, propagation loss of $3.1 \pm 0.3 \mathrm{~dB} / \mathrm{cm}$ was measured. Approximately $2 \mathrm{~dB} / \mathrm{cm}$ are due to silicon absorption loss at $\lambda \sim 7.7 \mu \mathrm{m}$. The remaining $\sim 1 \mathrm{~dB} / \mathrm{cm}$ loss is attributed to increased lateral leakage with respect to the simulated value and scattering loss caused by fabrication imperfections.

Table 3. Geometrical parameters and measured propagation loss at $\lambda \sim 7.7 \mu \mathrm{m}$.

\begin{tabular}{|c|c|c|c|c|c|}
\hline$H(\mu \mathrm{m})$ & $W(\mu \mathrm{m})$ & $W_{\text {clad }}(\mu \mathrm{m})$ & $L_{\mathrm{Si}}(\mu \mathrm{m})$ & $\Lambda(\mu \mathrm{m})$ & Measured propagation loss $(\mathrm{dB} / \mathrm{cm})$ \\
\hline 1.4 & 2.9 & 3 & 0.25 & 0.9 & $3.1 \pm 0.3$ \\
\hline
\end{tabular}

Bends were fabricated. $90^{\circ}$ bends are defined by the curvature radius $R$, while S-bends are characterized by their length $L$ and the offset $S$ between the input and output ports. SEM images of a $90^{\circ}$ bend is shown in Fig. 2(b). Final dimensional values are provided in Table 4. For both types of bends, loss smaller than $0.1 \mathrm{~dB} /$ bend was measured.

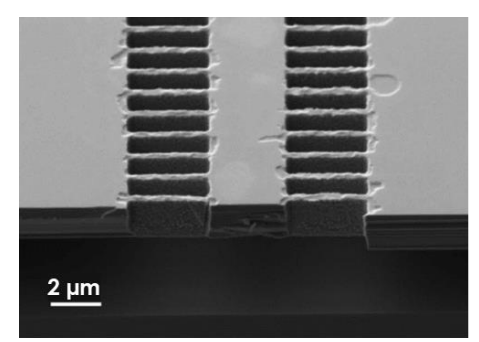

(a)

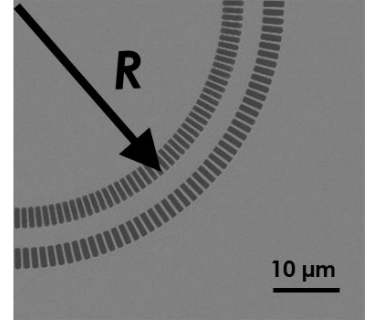

(b)

Figure 2. Scanning Electron Microscope (SEM) images of (a) cross-section of straight suspended waveguide, (b) top view of suspended $90^{\circ}$-bend and (c) top view of suspended S-bend.

Table 4. Geometrical parameters of $90^{\circ}$ - and S-bends and measured propagation loss at $\lambda \sim 7.7 \mu \mathrm{m}$.

\begin{tabular}{|c|c|c|c|c|}
\hline Type of bend & $R(\mu \mathrm{m})$ & $S(\mu \mathrm{m})$ & $L(\mu \mathrm{m})$ & Measured propagation loss $(\mathrm{dB} /$ bend $)$ \\
\hline $90^{\circ}$-bend & 35 & -- & -- & $<0.1$ \\
\hline S-bend & -- & 5 & 75 & $<0.1$ \\
\hline
\end{tabular}

\section{SUSPENDED SILICON WAVEGUIDES WITH SHIFTED BRAGG GRATING LATERAL CLADDING}

As an alternative, suspended silicon waveguides are designed with a shifted Bragg grating lateral cladding. Figure 3(a) depicts a schematic of a shifted Bragg suspended silicon waveguide. If the cladding periodicity is increased up to $\Lambda=\Lambda_{\text {Bragg }}=1.44 \mu \mathrm{m}$, the fundamental mode propagating through the waveguide is fully reflected, frustrating waveguiding. Fig 3(b) $(\Delta=0)$ shows the power transmission as a function of the wavelength. As it can be seen, in this case there is a band gap between $\lambda \sim 7.65 \mu \mathrm{m}$ and $\lambda \sim 7.70$. However, if both sides of the lateral cladding are shifted by a half period, i.e. $\Delta=\Lambda / 2=0.72 \mu \mathrm{m}$, with respect to each other, Bragg reflections at each discontinuity interfere destructively, enabling guiding of light as if the waveguide operated in the SWG regime. Intermediate shifts are also represented, showing that the band gap is reduced as the shift is increased. By using a shifted Bragg grating lateral cladding, the period $\Lambda$ is increased compared to standard suspended waveguides, thus enabling the utilization of longer lengths $L_{\mathrm{Si}}$ for the same duty cycle. 

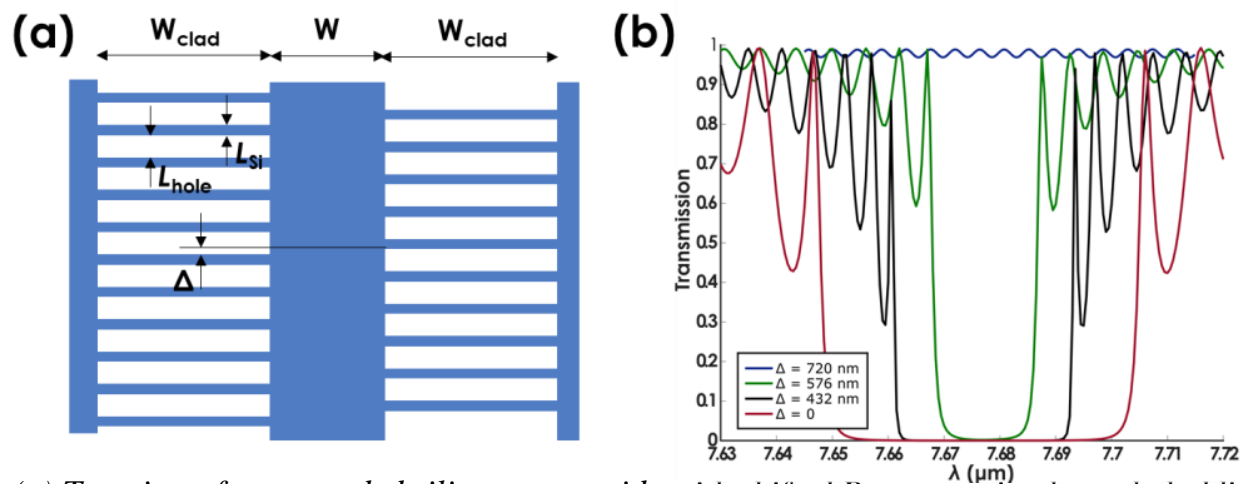

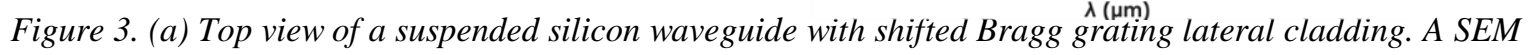
image of the fabricated structure is shown in the bottom part of the diagram. (b) Simulated power transmission as a function of the wavelength for different shifts $\Delta$.

Table 5 includes the geometrical parameters and the measured propagation loss of the shifted Bragg suspended waveguide. The pitch $\Lambda$ and the width of the silicon strips are increased up to $1.44 \mu \mathrm{m}$ (Bragg regime) and 300 $\mathrm{nm}$, respectively. We fabricated and characterized the waveguides using the same setup as in conventional suspended silicon waveguides. A propagation loss of $5.1 \pm 0.6 \mathrm{~dB} / \mathrm{cm}$ was measured. The increased loss compared to conventional SWG-cladding suspended silicon waveguides is attributed to a higher lateral leakage, as well as fabrication issues such as sidewall roughness.

Table 5. Geometrical parameters required for single-mode operation.

\begin{tabular}{|c|c|c|c|c|c|c|}
\hline$H(\mu \mathrm{m})$ & $W(\mu \mathrm{m})$ & $W_{\text {clad }}(\mu \mathrm{m})$ & $L_{\mathrm{Si}}(\mu \mathrm{m})$ & $\Lambda(\mu \mathrm{m})$ & $\Delta(\mu \mathrm{m})$ & Measured propagation loss $(\mathrm{dB} / \mathrm{cm})$ \\
\hline 1.4 & 2.9 & 4 & 0.3 & 1.44 & 0.72 & $5.1 \pm 0.6$ \\
\hline
\end{tabular}

\section{CONCLUSIONS}

In this work we have reviewed our latest progress in suspended silicon waveguides for the long-wavelength midinfrared band. Suspended waveguides with subwavelength grating and with shifted Bragg grating lateral cladding are demonstrated, with propagation loss of $3.1 \pm 0.3 \mathrm{~dB} / \mathrm{cm}$ and $5.1 \pm 0.6 \mathrm{~dB} / \mathrm{cm}$, respectively. Suspended silicon $90^{\circ}$ - and S-bends with less than $0.1 \mathrm{~dB} /$ bend are also reported.

\section{ACKNOWLEDGEMENTS}

We acknowledge funding from the Spanish Ministerio de Ciencia, Innovación y Universidad, Programa Estatal de Investigación, Desarrollo e Innovación Orientada a los Retos de la Sociedad (cofinanciado FEDER) (proyecto TEC2016-80718-R), the Spanish Ministerio de Ciencia, Innovación y Universidad, Programa Estatal de Promoción del Talento y su Empleabilidad en Investigación, Desarrollo e Innovación (ayuda FPU14/06121) and the Universidad de Málaga.

\section{REFERENCES}

[1] M. S. Vitiello et al.: Quantum cascade lasers: 20 years of challenges, Opt. Express, vol. 23, pp. 5167-5182, Feb. 2015.

[2] C. L. Tan and H. Mohseni: Emerging technologies for high performance infrared detectors, Nanophotonics, vol. 7, pp. 169-197, Oct. 2017.

[3] R. A. Soref et al.: Silicon waveguided components for the long-wave infrared region, J. Opt. A: Pure Appl. Opt., vol. 8, pp. 840-848, Aug. 2006.

[4] X. Chen et al.: The emergence of silicon photonics as a flexible technology platform, Proc. IEEE, vol. 106, pp. 2101-2116, Dec. 2018.

[5] S. A. Miller et al.: Low-loss silicon platform for broadband mid-infrared photonics, Optica, vol. 4, pp. 707712, Jul. 2017.

[6] Z. Cheng et al.: Mid-infrared suspended membrane waveguide and ring resonator on silicon-on-insulator, IEEE Phot. J., vol. 4, pp. 1510-1519, Oct. 2012.

[7] J. Soler Penadés et al.: Suspended silicon waveguides for long-wave infrared wavelengths, Opt. Lett., vol. 43, pp. 795-798, Feb. 2018.

[8] R. Halir et al., Subwavelength-grating metamaterial structures for silicon photonic devices, Proc. IEEE, vol. 106, pp. 2144-2157, Dec. 2018.

[9] P. Cheben et al.: Subwavelength integrated photonics, Nature, vol. 560, pp. 565-572, Aug. 2018. 